\title{
Scattering and radiation of water waves by permeable barriers
}

\author{
M. M. Lee and A. T. Chwang ${ }^{\text {a) }}$ \\ Department of Mechanical Engineering, The University of Hong Kong, Pokfulam Road, Hong Kong
}

(Received 5 October 1998; accepted 9 September 1999)

\begin{abstract}
The two-dimensional problems of scattering and radiation of small-amplitude water waves by thin vertical porous plates in finite water depth are considered using the linear water wave theory. Applying the method of eigenfunction expansion, these boundary value problems are converted to certain dual series relations. Solutions to these relations are then obtained by a suitable application of the least squares method. For the scattering problem, four different basic configurations of the barriers are investigated, namely, (I) a surface-piercing barrier, (II) a bottom-standing barrier, (III) a totally submerged barrier, and (IV) a barrier with a gap. The performance of these types of barriers as a breakwater are examined by studying the variation of their reflection and transmission coefficients, hydrodynamic forces and moments for different values of the porous effect parameter defined by Chwang [J. Fluid Mech. 132, 395-406 (1983)], or the Chwang parameter. For the radiation problem, three types of wavemakers, which resemble types (I), (II), and (III) of the above-mentioned configuration, are analyzed. The dependence of the amplitude to stroke ratio on other parameters is also investigated to study the features of these wavemakers. (C) 2000 American Institute of Physics. [S1070-6631(00)00201-4]
\end{abstract}

\section{INTRODUCTION}

The problems of scattering and radiation of water waves by vertical barriers have drawn the attention of many researchers since the early works of Ursell. ${ }^{1,2}$ Solutions of these problems are important in engineering applications such as breakwaters and wavemakers. In principle, twodimensional problems involving thin vertical barriers in deep water can be solved analytically in closed form with the reflection and transmission coefficients being expressed in terms of known functions or definite integrals. Because of the complexity of the problems, few explicit solutions have been derived. Numerical or semianalytical methods, such as the boundary integral equation method, the variational method, matched eigenfunction expansions and the Galerkin approximation, have to be used to obtain approximate solutions for problems of finite water depth.

For scattering problems, Dean ${ }^{3}$ used the complex variable technique to solve the submerged barrier problem. Ursell $^{1}$ utilized an integral equation procedure based on Havelock's expansion of the wave potential to obtain the solution. Williams ${ }^{4}$ exercised a simple reduction method to obtain the transmission and reflection coefficients without going into details of obtaining the velocity potentials. Evans ${ }^{5}$ applied the complex variable method to study the wave scattering problem of a completely submerged finite vertical plate in deep water. Tuck ${ }^{6}$ employed the method of matched asymptotic expansions to obtain an approximate transmission coefficient for a vertical wall with a gap. Porter ${ }^{7}$ by making use of the complex variable method as well as Green's integral theorem solved the problem of wave trans-

\footnotetext{
a) Telephone: (852) 2859-2634; Fax: (852) 2858-5415; electronic mail: atchwang@hkucc.hku.hk
}

mission through a gap in a vertical barrier in deep water.

Meanwhile, many researchers have engaged in generalizing and developing various methods from time to time to solve these problems in deep water, for example, Stoker, ${ }^{8}$ Levine and Rodemich, ${ }^{9}$ Lewin, ${ }^{10}$ and Mei. ${ }^{11}$

It is generally recognized that these problems in finite water depth do not have explicit solutions and thus numerical or semianalytical methods have been used for their solutions. A considerable number of works have been done by these methods such as Liu and Abbaspour, ${ }^{12}$ Losada et al., ${ }^{13}$ Abul-Azm, ${ }^{14}$ and Porter and Evans. ${ }^{15}$

For radiation problems, Ursell ${ }^{2}$ used the Havelock wavemaker theory to obtain an explicit solution for the wave radiation due to the small oscillation of a partially submerged vertical plate in water of infinite depth. Later, Evans ${ }^{16}$ solved both scattering and radiation problems for a completely submerged thin vertical plate in infinitely deep water.

Explicit solutions to these problems in finite water depth are extremely difficult to obtain. Evans and Linton ${ }^{17}$ used matched eigenfunction expansions to obtain the hydrodynamic characteristics of a submerged thin plate hinged about its point of attachment to the bottom of a constant depth fluid. Evans and Porter ${ }^{18}$ showed how to use a Galerkin approximation and a judicious choice of expansion functions to solve the radiation problem of a thin rolling plate in water of finite depth.

All the works mentioned above are involved with impermeable barriers. When a barrier is permeable, the problem has not received much attention in the literature. Using Green's integral theorem, Macaskill ${ }^{19}$ studied the wave scattering problem of a permeable thin barrier by converting it to integrodifferential equations. The equations were discretized and solved numerically for barriers consisting of disconnected arcs. However, no full solution was given for a barrier 
with uniform fine-pores. Chwang ${ }^{20}$ developed a porous wavemaker theory to study the generation of water waves by the harmonic oscillation of a thin porous plate, extended from the free surface to the channel bottom, in water of finite depth. Recently, Chwang and $\mathrm{Chan}^{21}$ gave a general review on the interaction between porous media and wave motion.

The objective of the present paper is to study the scattering and radiation of water waves by vertical permeable barriers in water of finite depth. The mathematical formulation for both scattering and radiation problems is presented in Sec. II. Numerical results on the transmission and reflection coefficients as well as the hydrodynamic forces and moments for various types of permeable barriers are given in Sec. III. Discussion on results and the effects of important parameters are also presented in Sec. III. Finally, conclusions are given in Sec. IV.

\section{MATHEMATICAL FORMULATION}

\section{A. Scattering problems}

Cartesian coordinates, as shown in Fig. 1, are chosen with the mean-free surface at $y=h$. The fluid, which occupies $0<y<h$ and $-\infty<x<\infty$, is divided into two regions: a negative region, $x<0$, with velocity potential $\Phi_{-}$and a positive region, $x>0$, with velocity potential $\Phi_{+}$. The porous barriers are located at

(a) Type I: $x=0,(h-a)<y<h$ (a surface-piercing barrier);

(b) Type II: $x=0,0<y<(h-a)$ (a bottom-standing barrier);

(c) Type III: $x=0,(h-a-d)<y<(h-a)$ (a totally submerged barrier);

(d) Type IV: $x=0,0<y<(h-a-d),(h-a)<y<h$ (a barrier with a gap).

In the following discussion, the barrier itself is denoted by $L_{b}$, and the gap is denoted by $L_{g}$, so that $L_{b} \cup L_{g}$ is $[0, h]$. The fluid is inviscid and incompressible, and the flow is irrotational. Under the assumption of the linear wave theory, the wave field is represented by a velocity potential $\Phi(x, y, t)$ which satisfies the Laplace equation,

$$
\nabla^{2} \Phi=0 .
$$

Because of the periodicity in time, the velocity potential $\Phi$ can be expressed as

$$
\Phi(x, y, t)=\phi(x, y) e^{-i \omega t}
$$

in which $\omega$ is the angular frequency.

The linearized boundary conditions for the spatial velocity potential $\phi(x, y)$ in the regions can be expressed as

(1) The free surface boundary condition,

$$
(\partial \phi / \partial y)-\left(\omega^{2} \phi / g\right)=0 \quad(\text { for } y=h) .
$$

(2) The bottom boundary condition,

$$
(\partial \phi / \partial y)=0 \quad(\text { for } y=0) \text {. }
$$

(3) The horizontal velocity and pressure must be continuous across the gap,

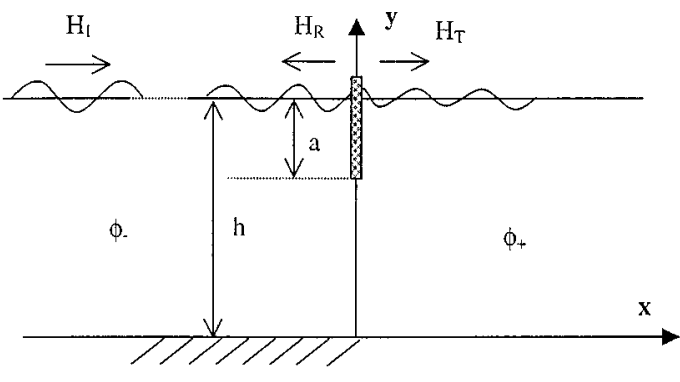

a) Type I: a surface-piercing porous barrier

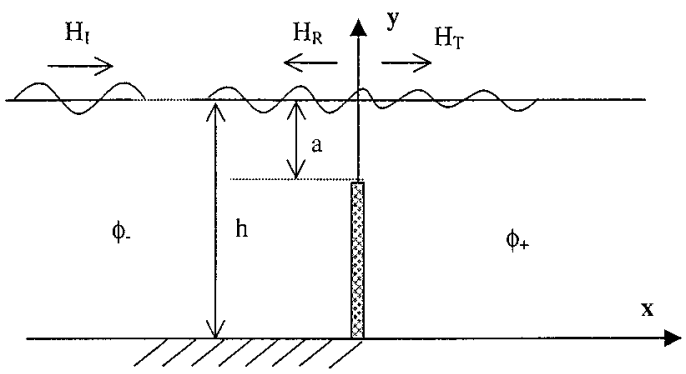

b) Type II: a bottom-standing porous barrier

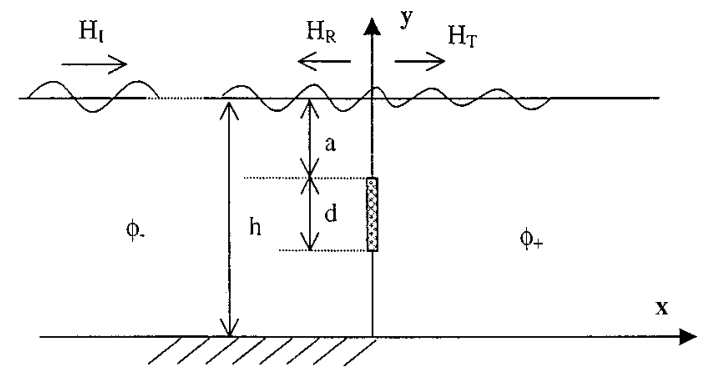

c) Type III: a totally submerged porous barrier

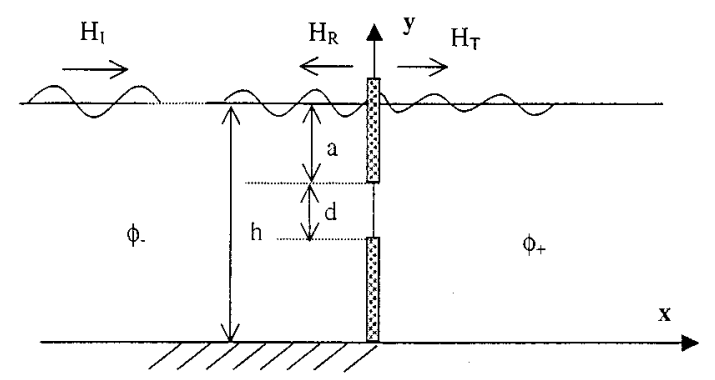

d) Type IV: a porous barrier with a single gap

FIG. 1. Definition sketch for four types of porous barriers. (a) Type I: a surface-piercing porous barrier. (b) Type II: a bottom-standing porous barrier. (c) Type III: a totally submerged porous barrier. (d) Type IV: a porous barrier with a single gap.

$$
\begin{aligned}
& \frac{\partial \phi_{-}}{\partial x}=\frac{\partial \phi_{+}}{\partial x} \text { on } x=0, \quad y \in L_{g}, \\
& \phi_{-}=\phi_{+} \text {on } x=0, \quad y \in L_{g} .
\end{aligned}
$$

(4) Let $W(y, t)$ be the normal velocity of the flow inside the porous barrier. According to Chwang, ${ }^{20} W(y, t)$ is linearly proportional to the pressure difference across the barrier, which is based on the assumption that the thin 
porous plate is made of material with fine and uniform pores so that the resistance effect dominates over the inertial effect and the flow through the plate is linearly proportional to the pressure difference between the two sides of the porous plate $\left(\right.$ Taylor $^{22}$ ),

$W(y, t)=\frac{b}{\mu}\left[P_{+}(0, y, t)-P_{-}(0, y, t)\right]$ on $x=0, y \in L_{b}$,

where $\mu$ is the coefficient of dynamic viscosity and $b$ is a material constant. Assuming that the hydrodynamic pressure $P$ and the porous flow $W$ are all periodic functions and have a time factor $e^{-i \omega t}$,

$W=w(y) e^{-i \omega t}$ and $P_{ \pm}=p_{ \pm}(x, y) e^{-i \omega t}$,

and relating the hydrodynamic pressure with the velocity potentials through the linearized Bernoulli equation, we have

$$
\begin{aligned}
w(y) & =\frac{b}{\mu}\left[p_{+}(0, y)-p_{-}(0, y)\right] \\
& =\frac{i \omega \rho b}{\mu}\left[\phi_{+}(0, y)-\phi_{-}(0, y)\right] \text { for } y \in L_{b} .
\end{aligned}
$$

The normal velocity on the porous barrier surface is related to the normal velocity of the flow inside it by

$$
\frac{\partial \phi_{-}}{\partial x}=\frac{\partial \phi_{+}}{\partial x}=-w(y) \text { on } x=0, y \in L_{b} .
$$

(5) At infinity, on both sides of the barrier, $x \rightarrow \pm \infty$, the scattered wave must be outgoing.

A train of regular, small-amplitude progressive waves of height $H_{I}$ and frequency $\omega$ propagates towards the barrier from the negative side. The incident waves are partially reflected with wave height $H_{R}$ at $x=0$ and partially transmitted with wave height $H_{T}$. The velocity potential of the incident waves is given by

$$
\phi_{I}=\frac{g H_{I}}{2 \omega} \frac{\cosh k_{0} y}{\cosh k_{0} h} e^{i k_{0} x}=\zeta_{I} \cosh k_{0} y \cdot e^{i k_{0} x},
$$

where $k_{0}$ is the wave number and is related to the angular frequency through the dispersion relation

$$
\omega^{2}=g k_{0} \tanh k_{0} h .
$$

An appropriate solution for the potentials, which satisfies (1)-(4), can be written as

$$
\phi_{-}=\phi_{I}+\sum_{n=0}^{\infty} A_{n} \cos \mu_{n} y \cdot e^{+\mu_{n} x}
$$

and

$$
\phi_{+}=\sum_{n=0}^{\infty} B_{n} \cos \mu_{n} y \cdot e^{-\mu_{n} x},
$$

where $\mu_{0}=-i k_{0}$ and $\mu_{n}=k_{n}$ for $n>0$ and $k_{n}$ are the positive real roots of the dispersion relation

$$
\omega^{2}=-g k_{n} \tan k_{n} h .
$$

The first term of (10a) represents the incident wave, and the summation terms represent the scattered waves. The coeffi- cients $A_{n}$ and $B_{n}$ are unknown complex numbers to be determined. It should be noted that $\cos \mu_{n} y(n=1,2, \ldots)$ forms a complete orthogonal set with

$$
\int_{0}^{h} \cos \mu_{n} y \cos \mu_{m} y \cdot d y=\delta_{n m} N^{2},
$$

where $\delta_{n m}$ is the Kronecker delta and

$$
N^{2}\left(\mu_{n}\right)= \begin{cases}\frac{\sinh 2 k_{0} h+2 k_{0} h}{4 k_{0}} & (\text { for } n=0), \\ \frac{\sin 2 k_{n} h+2 k_{n} h}{4 k_{n}} & (\text { for } n>0) .\end{cases}
$$

By using the condition that the horizontal velocity, $(\partial \phi / \partial x)$, is continuous along $x=0$ and making use of the fact that the $\cos \mu_{n} y$ functions are orthogonal, it can be shown that

$$
A_{0}+B_{0}=1 \text { and } A_{n}+B_{n}=0 .
$$

Substituting (10) and (12) into (7), we have

$$
\begin{aligned}
\frac{\partial \phi_{-}}{\partial x}=\frac{\partial \phi_{+}}{\partial x} & =i k_{0} \phi_{I}(0, y)+\sum_{n=0}^{\infty} \mu_{n} A_{n} \cos \mu_{n} y \\
& =-w(y) \text { for } y \in L_{b} .
\end{aligned}
$$

By Eqs. (6c), (10), and (12),

$$
w(y)=-i k_{0} G_{0}\left[2 \sum_{n=0}^{\infty} A_{n} \cos \mu_{n} y\right] \text { for } y \in L_{b},
$$

where $G_{0}=\left(\rho b \omega / \mu k_{0}\right)$ is the porous effect parameter defined by Chwang, ${ }^{20}$ or the Chwang parameter.

The continuity of pressure along the gap now gives

$$
p_{+}(0, y)-p_{-}(0, y)=0
$$

or

$$
2 \sum_{n=0}^{\infty} A_{n} \cos \mu_{n} y=0 \quad \text { for } y \in L_{g}
$$

A dual series relation $\left(\right.$ Sneddon $\left.^{23}\right)$ can be constructed by rewriting (13) and (14) as

$$
\begin{aligned}
& i k_{0} \phi_{I}(0, y)+\sum_{n=0}^{\infty}\left(\mu_{n}-2 i k_{0} G_{0}\right) A_{n} \cos \mu_{n} y=0 \\
& \qquad \begin{array}{r}
\text { for } y \in L_{b}, \\
2 \sum_{n=0}^{\infty} A_{n} \cos \mu_{n} y=0 \quad \text { for } y \in L_{g} .
\end{array}
\end{aligned}
$$

The solution of this dual series relation can be obtained approximately by the least squares method, which requires to minimize the value of the integral,

$$
\left\|S_{N}-f\right\|=\int_{0}^{h}\left|S_{N}(y)-f(y)\right|^{2} d y,
$$

with respect to $A_{n}$. In Eq. (16) the functions $S_{N}(y)$ and $f(y)$ are given by 


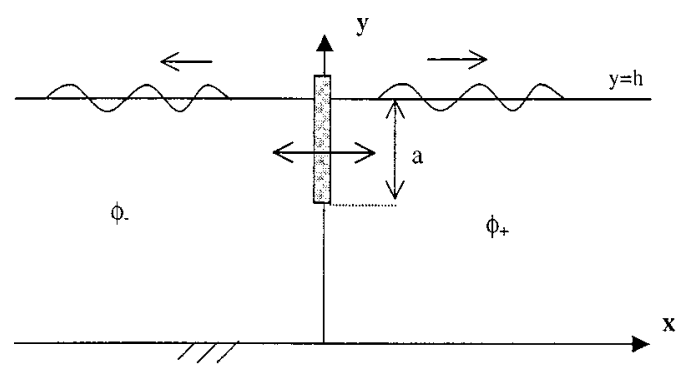

a) Type I: a surface-piercing porous wavemaker

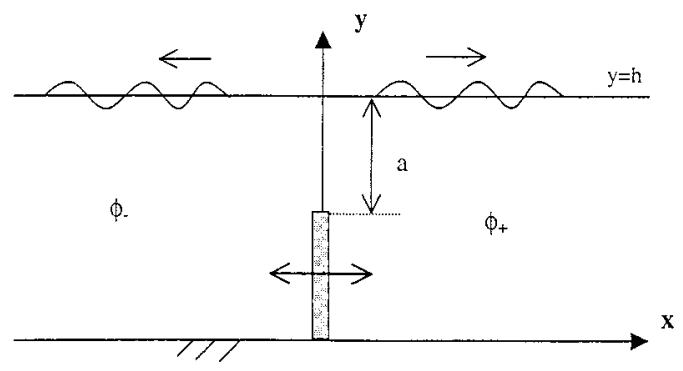

b) Type II: a bottom-standing porous wavemaker

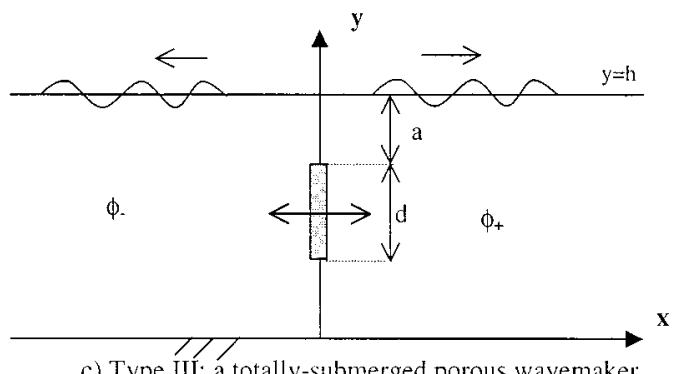

c) Type III: a totally-submerged porous wavemaker

FIG. 2. Definition sketch for three types of porous wavemakers. (a) Type I: a surface-piercing porous wavemaker. (b) Type II: a bottom-standing porous wavemaker. (c) Type III: a totally submerged porous wavemaker.

$$
S_{N}(y)=\left\{\begin{array}{l}
\sum_{n=0}^{N}\left(\mu_{n}-2 i k_{0} G_{0}\right) A_{n} \cos \mu_{n} y \quad \text { for } y \in L_{b} \\
\sum_{n=0}^{N} A_{n} \cos \mu_{n} y \quad \text { for } y \in L_{g}
\end{array}\right.
$$

and

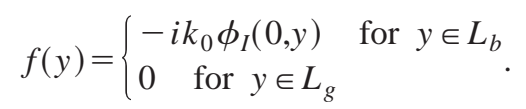

Therefore,

$$
\int_{0}^{h}\left[S_{N}-f\right] * \frac{\partial S_{N}(y)}{\partial A_{n}} d y=0, \quad(n=0,1,2, \ldots),
$$

where the superscript $*$ denotes the complex conjugate. An approximate solution can be obtained by truncating the infinite series to a finite series of $N$ terms. There are $N$ simultaneous equations with $N$ unknowns. A complex matrix equation of rank $N$ can be obtained, which can then be solved to obtain the values of $A_{n}$.

\section{B. Radiation problems}

As shown in Fig. 2, a vertical porous plate is forced to oscillate horizontally with an angular frequency $\omega$ and a small displacement $s_{0}$ about the $x=0$ axis. This oscillating porous plate generates outgoing water waves. Cartesian coordinates are chosen with the mean free surface at $y=h$. The studying domain, which occupies $0<y<h$ and $-\infty<x$ $<\infty$, is divided into two regions; a left-hand region, $x<0$, with velocity potential $\Phi_{-}$, and a right-hand region, $x>0$, with velocity potential $\Phi_{+}$. The plates occupy the following positions, respectively,

(a) Type I: $x=0$ and $(h-a)<y<h$ (a surface piercing wavemaker);

(b) Type II: $x=0$ and $0<y<(h-a)$ (a bottom-standing wavemaker);

(c) Type III: $x=0$ and $(h-a-d)<y<(h-a)$ (a totally submerged wavemaker).

The wavemaker, which has a horizontal velocity $U_{0}(y, t)=-i \omega s_{0} e^{-i \omega t}$, generates outgoing waves on both sides. Boundary conditions (3)-(6) are still valid. However, Eq. (7) should be replaced by

$$
\frac{\partial \phi_{-}}{\partial x}(0, y)=\frac{\partial \phi_{+}}{\partial x}(0, y)=-i \omega s_{0}-w(y) \text { for } y \in L_{b} .
$$

At infinity, $x \rightarrow \pm \infty$, the radiation wave must be outgoing, and the spatial velocity potentials of the generated waves can be given by

$$
\phi= \pm \frac{\omega H_{\text {out }}}{2 k_{0}} \frac{\cosh k_{0} y}{\sinh k_{0} y} e^{ \pm i k_{0} x} .
$$

The general solutions for the velocity potentials in the two regions satisfying the continuity of normal velocity, $\partial \phi / \partial x$, along $x=0$ are

$$
\begin{aligned}
& \phi_{+}=A_{0} \cosh k_{0} y e^{i k_{0} x}+\sum_{n=1}^{\infty} A_{n} \cos k_{n} y e^{-k_{n} x}, \\
& \phi_{-}=-A_{0} \cosh k_{0} y e^{-i k_{0} x}-\sum_{n=1}^{\infty} A_{n} \cos k_{n} y e^{k_{n} x},
\end{aligned}
$$

where $k_{0}$ and $k_{n}$ satisfy the dispersion relations

$$
\omega^{2}=g k_{0} \tanh k_{0} h=-g k_{n} \tan k_{n} h .
$$

The pressure in both regions must match at the gap. Hence by $(5 b)$,

$$
A_{0} \cosh k_{0} y+\sum_{n=1}^{\infty} A_{n} \cos k_{n} y=0 \quad \text { for } y \in L_{g} .
$$

By Eqs. (6c), (19), and (21), we have

$$
\begin{aligned}
& \left(-i k_{0}-2 i k_{0} G_{0}\right) A_{0} \cosh k_{0} y+\sum_{n=1}^{\infty}\left(k_{n}-2 i k_{0} G_{0}\right) A_{n} \cos k_{n} y \\
& =i \omega s_{0} \text { for } y \in L_{b} .
\end{aligned}
$$

Equations (22) and (23) are known as dual series relations. In order to determine the values of the coefficients, the relations must be solved simultaneously. The least squares method is used to minimize the following integral with respect to the unknown coefficients: 


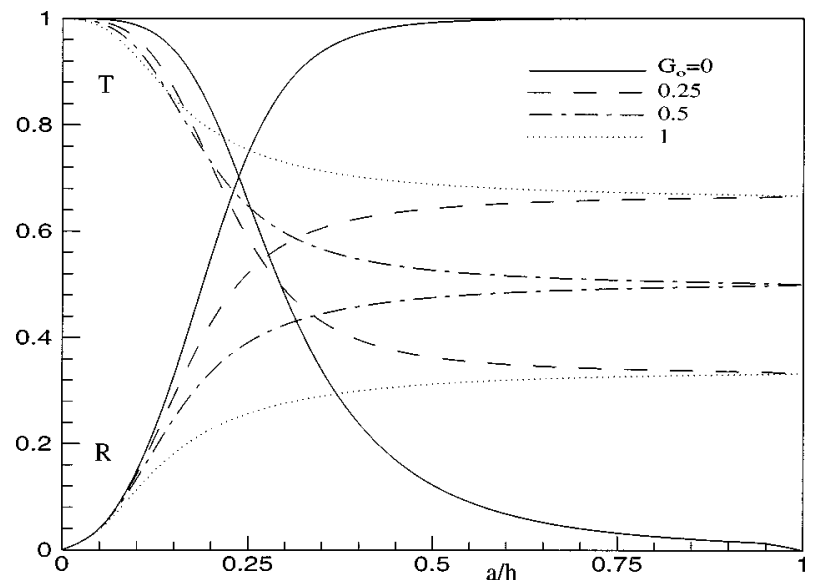

FIG. 3. $T$ and $R$ vs the dimensionless submergence depth $a / h$ for $h / \lambda$ $=0.44$, Type $\mathrm{I}$.

$$
\left\|S_{N}-f\right\|^{2}=\int_{0}^{h}\left|S_{N}-f(y)\right|^{2} d y,
$$

where $S_{N}$ is given by (17a) and

$$
f(y)=\left\{\begin{array}{l}
i \omega s_{0} \text { for } y \in L_{b} \\
0 \text { for } y \in L_{g}
\end{array} .\right.
$$

The results are

$$
\int_{0}^{h}\left[S_{N}-f\right] * \frac{\partial S_{N}(y)}{\partial A_{n}} d y=0, \quad(n=0,1,2, \ldots),
$$

where the superscript $*$ denotes the complex conjugate. There are $N$ simultaneous equations with $N$ unknowns, a complex matrix equation with dimension $N \times N$ can be obtained, which can then be solved to obtain the values of $A_{n}$ (Losada et al. ${ }^{13}$ ).

It can be noticed that the general form of the resultant dual series relations, (22) and (23), is similar to those given in Sec. II A. In fact, the results of the wave scattering problem can also be obtained by considering a special forcing function with the horizontal velocity distribution on the barrier opposite to that of the incident waves. This concept enlightens us on considering the problem of an active breakwater or active wave control. Although in this paper only the scattering and radiation problems of porous barriers are considered, the results revealing the performance of these three types of barriers are still useful to the preliminary understanding of the mechanisms involved in active wave control problems.

\section{RESULTS AND DISCUSSION}

The method of solution described above has been applied to solve certain typical cases of scattering and radiation problems. It seems appropriate and worthwhile at this point to mention that the method of least squares approximation for dual trigonometric series was first developed by Kelman and Koper $^{24}$ to obtain solutions for general dual trigonometric equations. The convergence of the dual orthogonal series in Hilbert space was later established by Feinerman and Kelman. ${ }^{25}$ This provides the theoretical background to apply

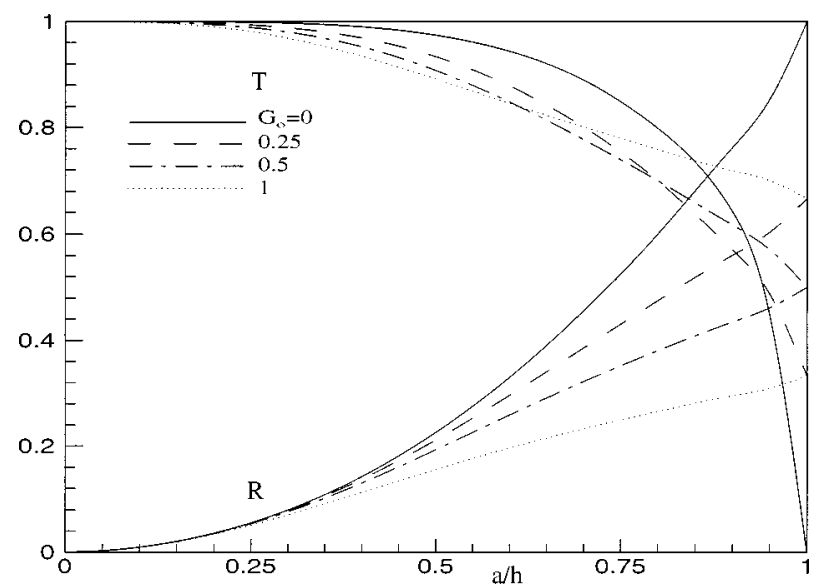

FIG. 4. $T$ and $R$ vs the dimensionless submergence depth $a / h$ for $h / \lambda$ $=0.12$, Type I.

the method to the present problem. Moreover, since the theory is applicable to all square integrable functions, the method can automatically deal with the square-root singularity at the tip of the thin barrier.

The problem of convergence has been carefully taken into account by examining certain test cases. Based on the test results, the convergence of the solution depends on the geometry and configuration of the barrier. By considering the accuracy and computational time consumption, appropriate value of $N$ has been chosen for each particular case considered in the present paper.

The performance of the four types of porous barriers used as breakwaters is discussed by considering the variation of their transmission and reflection coefficients. The transmission coefficient $T$ is defined as the ratio of the maximum wave height of the transmitted waves to that of the incident wave (see Fig. 1),

$$
T=\frac{H_{T}}{H_{I}},
$$

whereas the reflection coefficient is defined as the ratio of the maximum wave height of the reflected waves to that of the incident wave,

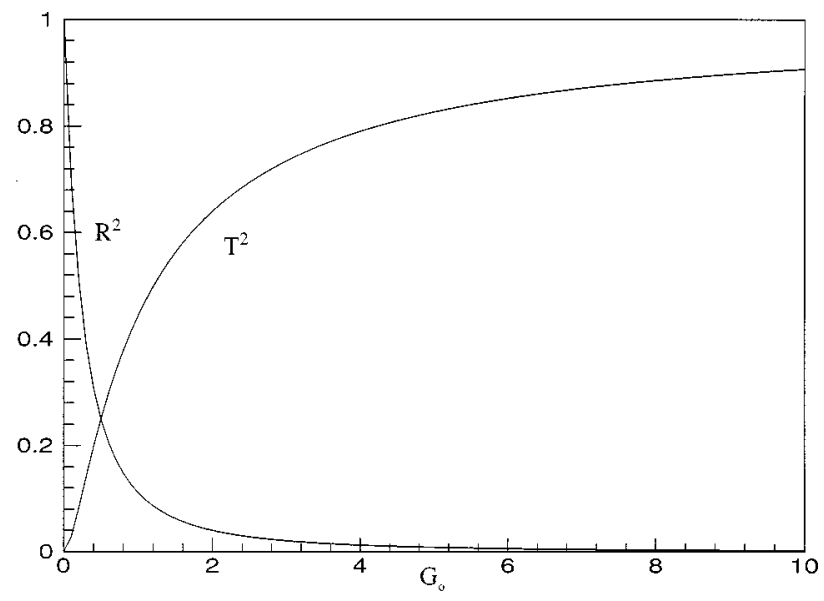

FIG. 5. $T$ and $R$ vs the Chwang parameter $G_{0}=\rho \omega b / \mu k_{0}$. 


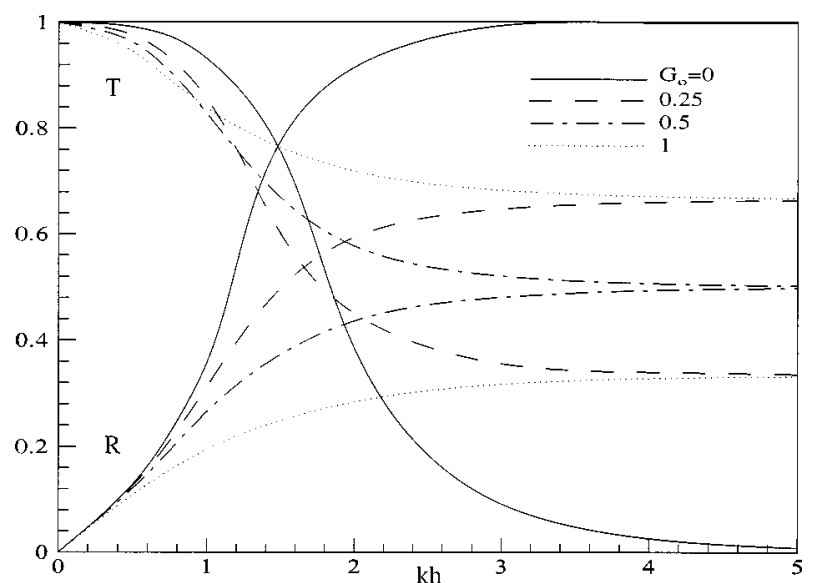

FIG. 6. $T$ and $R$ vs $k h$ with $a / h=0.5$, Type I.

$$
R=\frac{H_{R}}{H_{I}} .
$$

The characteristics of the three types of wavemakers are also examined by calculating their amplitude to stoke ratios.

\section{A. Scattering problems}

\section{A surface-piercing barrier}

Figures 3 and 4 show the variations of the transmission coefficient $T$ and the reflection coefficient $R$ for different values of the Chwang parameter, $G_{0}$, and different values of water depth. Figure 3 is a graph for the case of dimensionless water depth $h / \lambda=0.44$, which in fact corresponds to a deep water case. It can be seen that, when $a / h=0$, all the $T$-curves (curves for the transmission coefficients) start from 1 while all the $R$-curves (curves for the reflection coefficients) start from 0 . That means when there is no barrier, all the incident waves are transmitted to the other side and there is no reflection. When $a / h$ is increased, the $T$-curves drop rapidly and $R$-curves rise accordingly. This observation agrees well with Ursell's ${ }^{1}$ result, which states that for deep water waves most of the energy is confined near the free surface, so the transmitted wave energy decreases drastically when the parameter, $2 \pi a / \lambda$, is greater than one. When $a / h=1$, that means

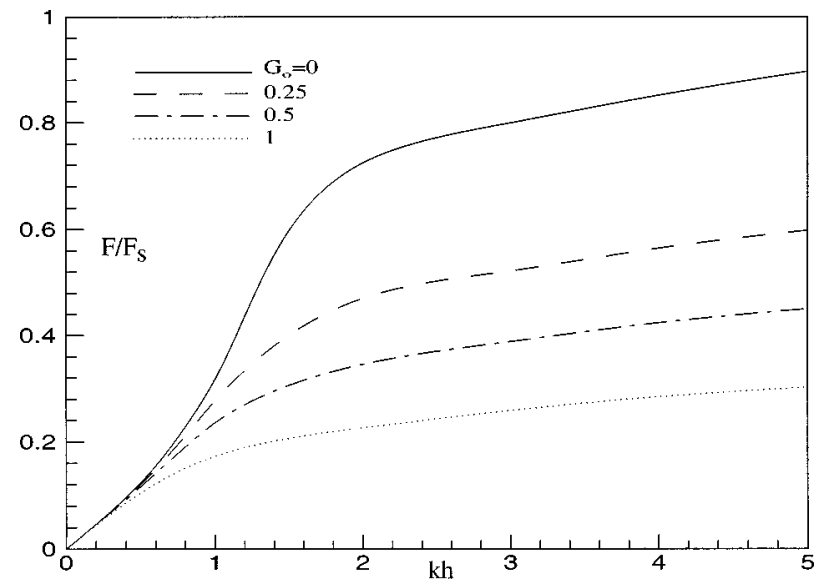

FIG. 7. Horizontal force $\left(F / F_{s}\right)$ vs $k h$ with $a / h=0.5$, Type I.

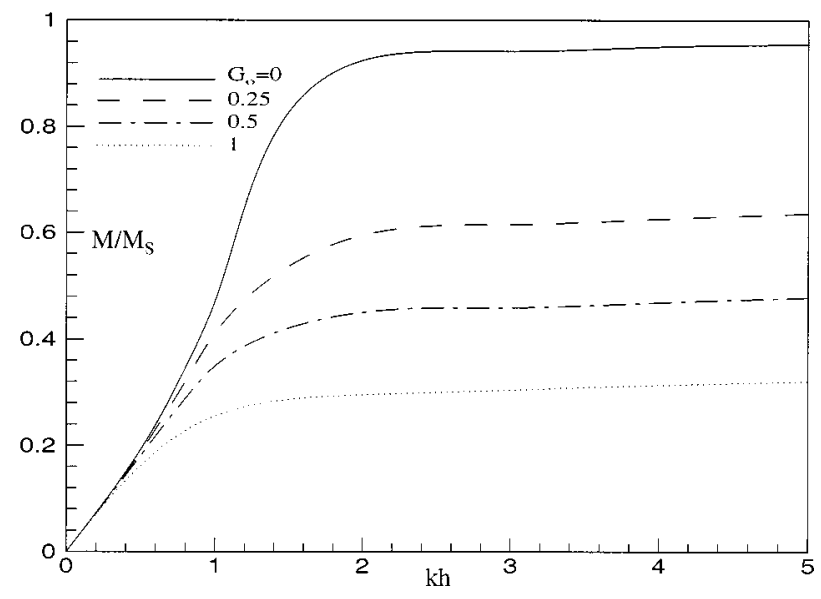

FIG. 8. Overturning moment $\left(M / M_{s}\right)$ vs $k h$ with $a / h=0.5$, Type I.

the barrier is fully extended to the channel's bottom, the incident waves are totally or partially reflected depending on the values of the Chwang parameter $G_{0}$. Figure 5 shows the variation of $T$ and $R$ vs $G_{0}$ for these fully extended barriers, actually this result is the same as those given by Chwang and Dong. ${ }^{26}$ In general, the higher the values of $G_{0}$, the lower the values of the end point of $R$-curves, which implies more waves can be transmitted through the porous barrier to the other side for higher porosity factor. The general shape of the $R$-curves for different values of $G_{0}$ are of similar nature, but seems to be compressed downwards. This result can be comprehended with the physical intuition that when $G_{0}$ increases, the permeability is increased, which will allow more fluid and energy to pass through the barrier; and thus the reflection coefficients decrease.

In Fig. 4, the dimensionless water depth $h / \lambda$ is decreased to 0.12 , which corresponds to a shallow water case. It can be seen that the general shapes of the curves are different from those of the previous case. The result reveals that the barrier needs to be immersed more deeply into the water in order to obtain the same reflection coefficient. This is because the wave energy is more evenly distributed over the channel. In general, the effect of a porous barrier is to reduce

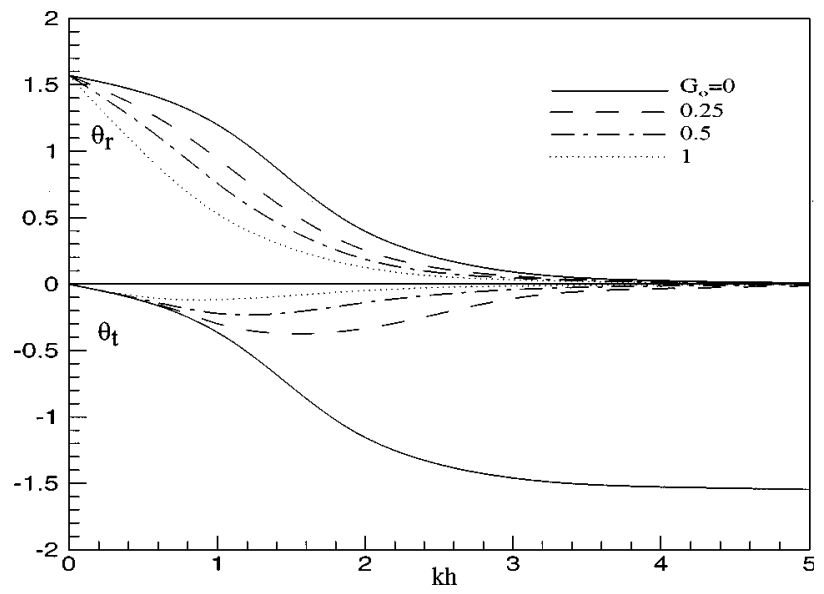

FIG. 9. Phase angles $\theta_{t}$ and $\theta_{r}$ vs $k h$ with $a / h=0.5$, Type I. 


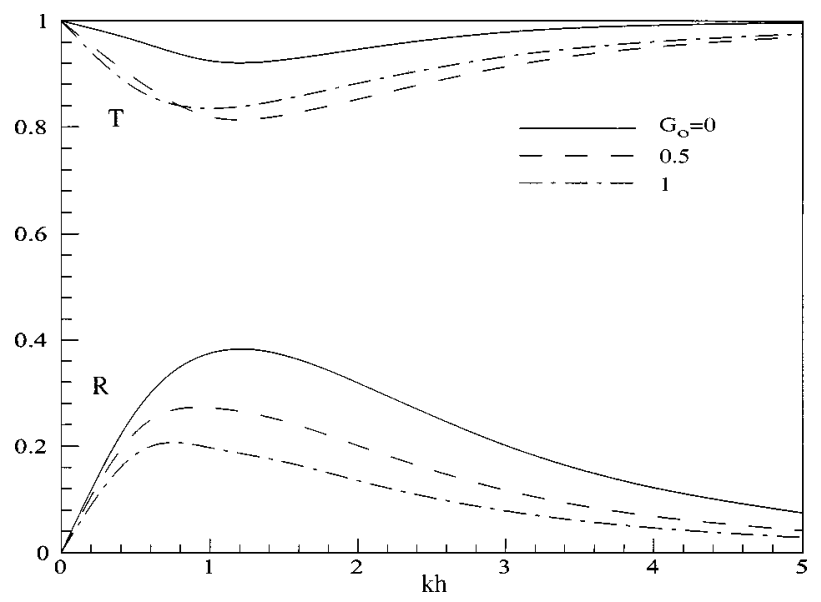

FIG. 10. $T$ and $R$ vs $k h$ with $a / h=0.25$, Type II.

the reflection coefficient and to cause the $R$-curves to move downwards.

It should be noted that when $G_{0}=0$, there is no energy loss in the barrier. Therefore, by conservation of energy, $R^{2}+T^{2}=1$. However, when $G_{0}>0$, some of the wave energy, depending on the value of the porosity (see Fig. 5), would be dissipated by the barrier, that means $R^{2}+T^{2}<1$. On the other hand, when the porosity of a barrier is increased, the permeability of the barrier is increased correspondingly. As the flow passing through the barrier increases, more wave energy can be transmitted through the barrier to the other side and thus causing the reflection coefficient $R$ to decrease. As a result, it is possible for two $T$-curves with different values of $G_{0}$ to intersect each other, while the corresponding $R$-curves do not intersect each other.

In fact, there are other important design-factors in regard to breakwaters. They are given as follows:

(1) The dimensionless hydrodynamic pressure force $F / F_{s}$,

$$
\begin{aligned}
\frac{F}{F_{s}} & =\frac{1}{F_{s}} \int_{h-a}^{h}\left[p_{-}(0, y)-p_{+}(0, y)\right] d y \\
& =\frac{1}{F_{s}} \int_{h-a}^{h} \rho\left[\frac{\partial \phi_{+}(0, y)}{\partial t}-\frac{\partial \phi_{-}(0, y)}{\partial t}\right] d y,
\end{aligned}
$$

where $F_{s}$ is the force per unit width for the full reflection case.

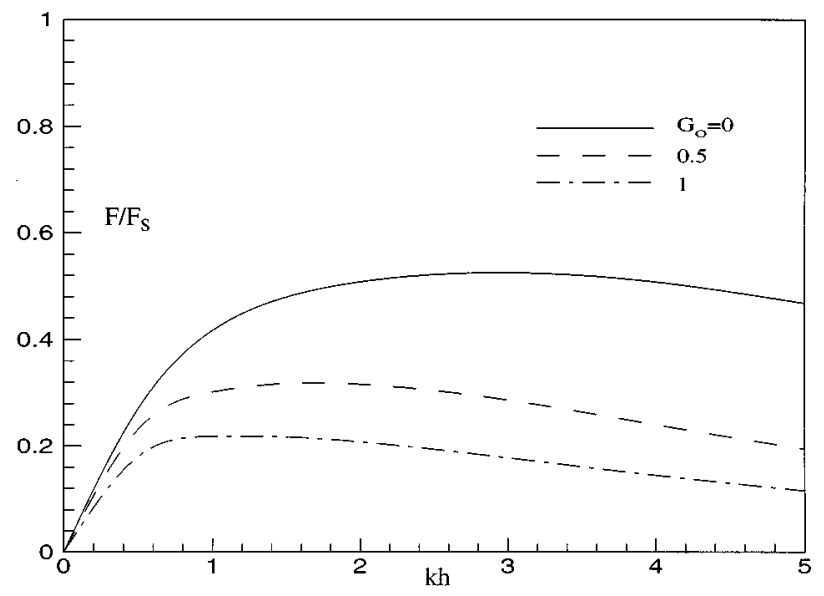

FIG. 11. Horizontal force $\left(F / F_{s}\right)$ vs $k h$ with $a / h=0.25$, Type II.

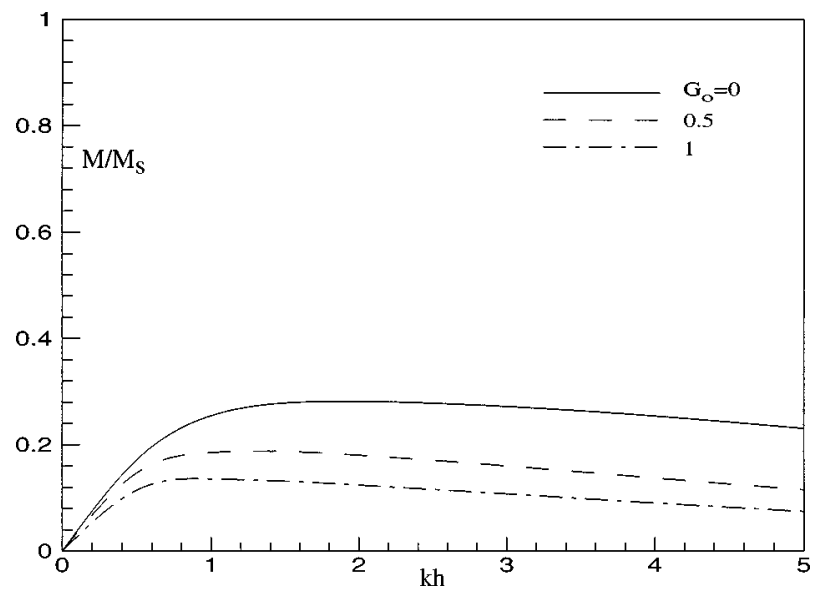

FIG. 12. Overturning moment $\left(M / M_{s}\right)$ vs $k h$ with $a / h=0.25$, Type II.

(2) The dimensionless overturning moment $M / M_{s}$,

$$
\begin{aligned}
\frac{M}{M_{s}} & =\frac{1}{M_{s}} \int_{h-a}^{h} y\left[p_{-}(0, y)-p_{+}(0, y)\right] d y \\
& =\frac{1}{M_{s}} \int_{h-a}^{h} \rho y\left[\frac{\partial \phi_{+}(0, y)}{\partial t}-\frac{\partial \phi_{-}(0, y)}{\partial t}\right] d y,
\end{aligned}
$$

where $M_{s}$ is the moment per unit width for the full reflection case.

(3) The phase angle of the transmitted and reflected waves, $\theta_{t}$ and $\theta_{r}$.

Figures 6-9 show the variation of the parameters, $T$ and $R, F / F_{s}, M / M_{s}, \theta_{t}$ and $\theta_{r}$, vs the dimensionless water depth $k h$ for different values of $G_{0}$. It can be observed that these parameters rise or change quickly when $k h$ is increased from 0 to 2 . This observation agrees with the physical intuition that a surface-piercing barrier is not so good to obstruct long waves. Long waves can pass through the barrier without too much reflection. However, when $k h$ is large, which means that the water depth is large relative to the wavelength, most of the incoming waves are reflected by the surface-piercing barrier. Therefore, all the parameters change rapidly from $k h=0$ to $k h=2$. The porous effect in general is to reduce the values of different parameters.

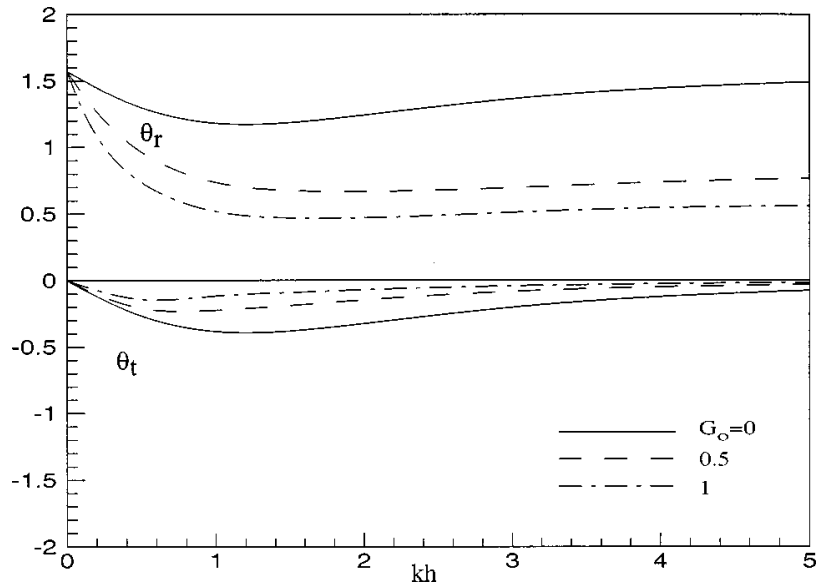

FIG. 13. Phase angles $\theta_{t}$ and $\theta_{r}$ vs $k h$ with $a / h=0.25$, Type II. 


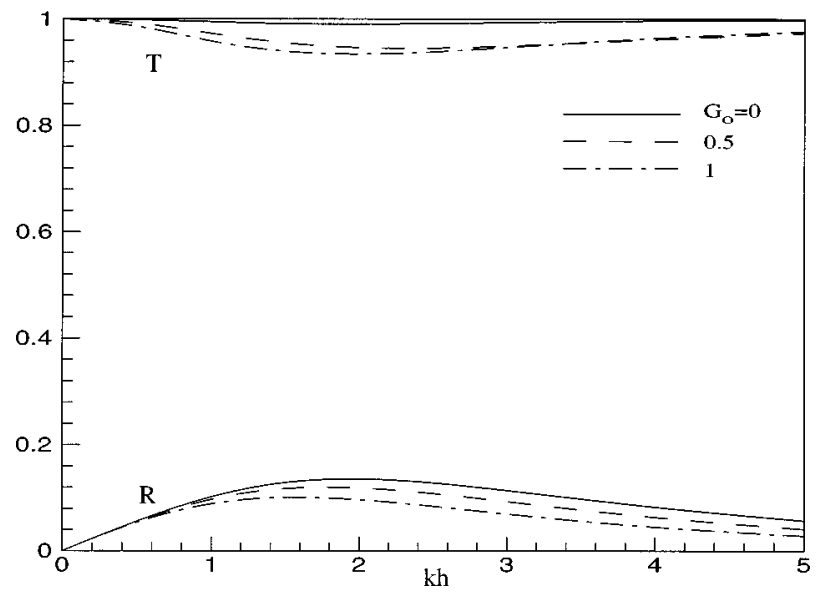

FIG. 14. $T$ and $R$ vs $k h$ for $a / h=0.25$ and $d / h=0.5$, Type III.

\section{A bottom-standing barrier}

Figures 10-13 show the variations of the different parameters vs the dimensionless water depth, $k h$, for the specific case of a bottom-standing barrier when $a / h=0.25$. It is interesting to note from Fig. 10 that the $R$-curves increase in the earlier stage, but when they reach certain maximum turning points they begin to decrease. This observation can be explained by the following argument. When the value of $k h$ is very small, it means that the wavelength of the incoming waves is very long. These long waves are almost totally transmitted. When $k h$ increases, the effect of the barrier begins to operate and thus the reflection coefficient $R$ increases. But when the value of $k h$ becomes large, it means that the wavelength is short, the effectiveness of the barrier decreases as the wave motion is now confined near the free surface. Similar observation can be found in graphs for the horizontal force, $F$, and the overturning moment, $M$, but the curves now decrease much slower after the turning points. Increase in porosity of the barrier, in general, reduces the reflection $R$, the horizontal force $F$, the overturning moment and the difference between the phase angles $\theta_{t}$ and $\theta_{r}$.

\section{A totally-submerged barrier}

Figure 14 shows the variation of $T$ and $R$ vs the dimensionless water depth $k h$ for $a / h=0.25$ and $d / h=0.5$. In this

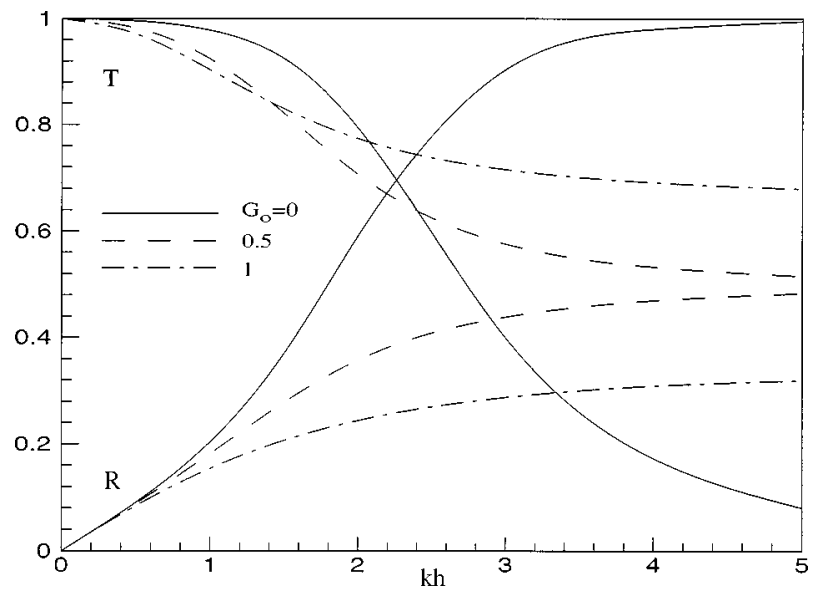

FIG. 15. $T$ and $R$ vs $k h$ for $a / h=0.3$ and $d / h=0.4$, Type IV.

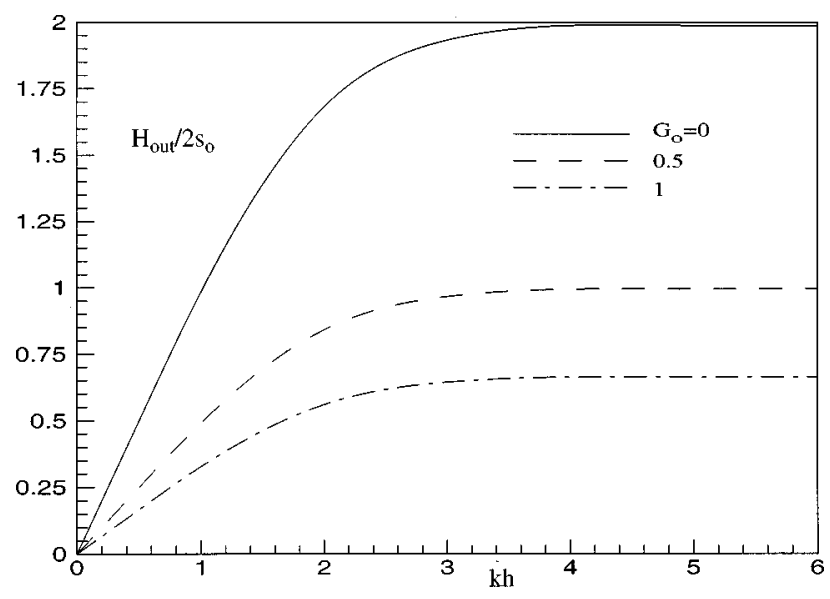

FIG. 16. Amplitude to stroke ratio vs $k h$ for a porous wavemaker.

figure, it can be seen that this barrier is not good for both shallow and deepwater cases. The optimal performance occurs for the case of intermediate water depth. It should be noted that the $R$-curves drop when the water depth $k h$ is greater than 2. This observation is similar to the results for a type II barrier in Fig. 10. It is remarkable, as compared with the results of the type II barrier in Fig. 10 that for the same barrier length the effectiveness of a type II barrier is better than that of a type III barrier. In general, an increase in the porosity of the barrier can reduce the reflection coefficient $R$.

\section{A barrier with a single gap}

Figure 15 shows how $T$ and $R$ vary with the dimensionless water depth $k h$ with $a / h=0.3$ and $d / h=0.4$. The general shape of the curves is similar to that of a surface-piercing barrier given in Fig. 6. When $k h$ is smaller than 3, which means the wavelength of the incident waves is much larger than the water depth, most of the incident waves can pass through the barrier's slit and thus the transmission is high. But, when $k h$ is large, most of the wave energy of the incident waves is now confined near the free surface, which is then obstructed by the upper portion of the barrier and reflected back. Therefore, the transmission is low. In general, increasing the porosity of a barrier can reduce the wave reflection.

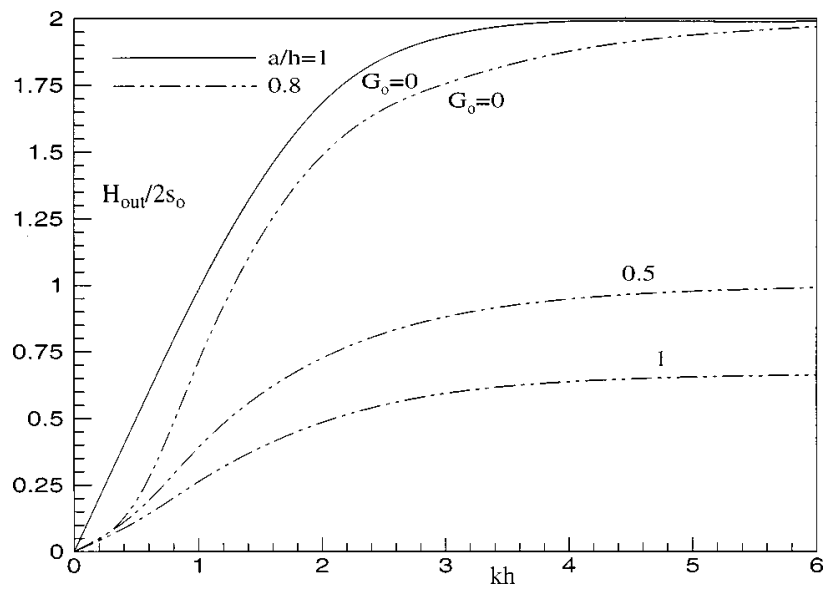

FIG. 17. Amplitude to stroke ratio vs $k h$ for different values of the dimensionless submergence $a / h$ with $G_{0}=0,0.5,1$, Type I wavemaker. 


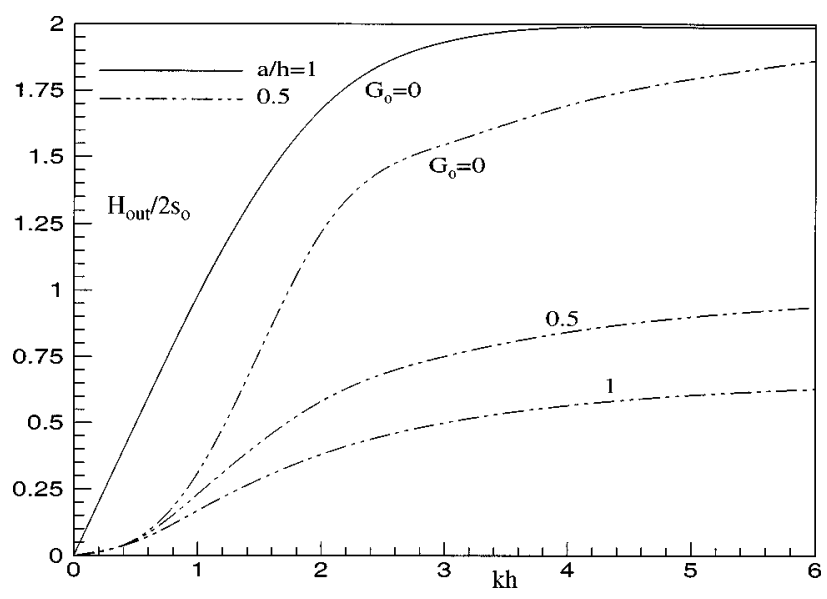

FIG. 18. Amplitude to stroke ratio vs $k h$ for different values of the dimensionless submergence $a / h$ with $G_{0}=0,0.5,1$, Type I wavemaker.

\section{B. Radiation problems}

\section{A surface-piercing wavemaker}

The effectiveness of this type of wavemaker is examined by studying the variation of the amplitude to stroke ratios with respect to the dimensionless water depth, $k h$, for different values of the Chwang parameter, $G_{0}$. Figure 16 shows how the amplitude to stroke ratios change with the water depth for the case of a piston-type porous wavemaker, which has been studied theoretically by Chwang. ${ }^{20}$ The results shown are obtained by the present method as a limiting case, which is in good agreement with the theoretical solution.

From Figs. 17-19, it can be seen clearly how the depth of submergence and the porosity of a type I wavemaker affect the performance of the corresponding wavemaker. It is remarkable to note that the behavior of the surface-piercing wavemaker with $a / h=0.5$ is just slightly poorer than that of the fully extended one. When $k h$ is small, it means that the wavemaker is oscillating very slowly and thus the long waves are generated; whereas when $k h$ is large, it means for the case of high frequency short waves. Not surprisingly, the amplitude to stroke ratios are reduced if we decrease the depth of submergence of the surface-piercing wavemaker. In

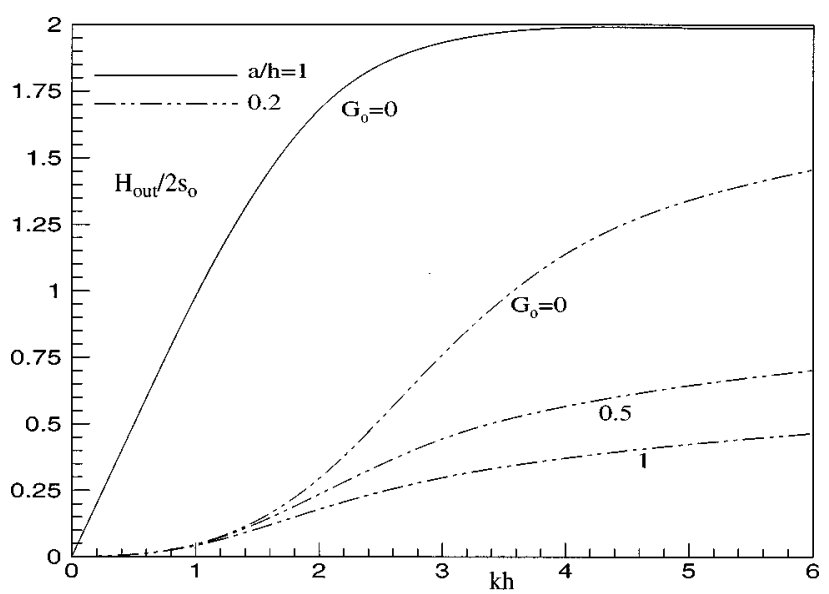

FIG. 19. Amplitude to stroke ratio vs $k h$ for different values of the dimensionless submergence $a / h$ with $G_{0}=0,0.5,1$, Type I wavemaker.

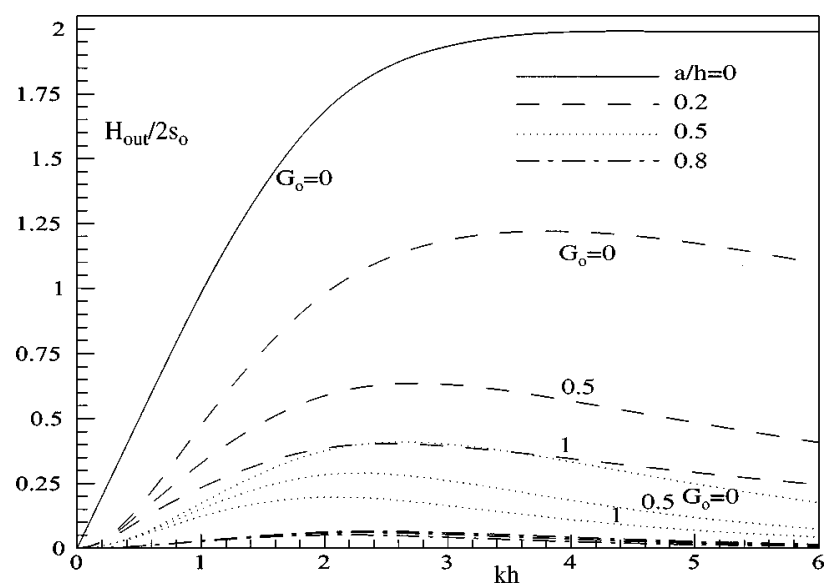

FIG. 20. Amplitude to stroke ratio vs $k h$ for different values of the dimensionless gap length $a / h$ with $G_{0}=0,0.5,1$, Type II wavemaker.

physical terms, this can be easily explained by the fact that since the forcing plate is now smaller, it can only excite smaller amount of water. Moreover, it should be noted that all the curves with $G_{0}=0$ seem to converge to 2 when $k h$ tends to infinity. This result suggests that the effectiveness to produce high frequency short waves with a surface-piercing wavemaker, whether it is slightly or deeply immersed, is generally good.

These figures also present how the Chwang parameter, $G_{0}$, affects the variation of the amplitude to stroke ratios for different settings of surface-piercing wavemakers. In these figures, it can be observed that increasing the Chwang parameter, $G_{0}$, results in smaller amplitude to stroke ratios. Not unexpectedly, larger $G_{0}$ means higher permeability and larger permeability will allow more fluid to pass through the barrier, which means that the forced oscillation of the wavemaker exert lesser force on the fluid and thus smaller amount of water gets excited. The general behavior of the curves in these three different cases, $a / h=0.8,0.5,0.2$ seems to be similar. All the curves for the impermeable wavemakers $G_{0}=0$ seem to converge to 2 . While the curves for $G_{0}$ $=0.5$ and $G_{0}=1$ seem to converge to 1 and $2 / 3$, respectively

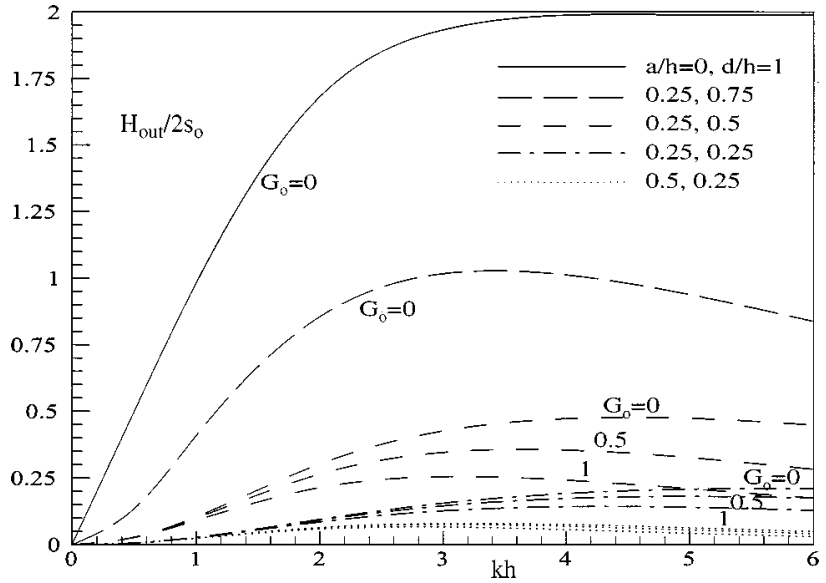

FIG. 21. Amplitude to stroke ratio versus $k h$ for different values of the dimensionless position $a / h$ and plate length $d / h$ with $G_{0}=0,0.5,1$, Type III wavemaker. 
which are the corresponding limiting values for the case of fully extended porous wavemakers. These results are equivalent to saying that the effectiveness of a surface-piercing porous wavemaker is as good as that of a fully extended porous wavemaker in producing high frequency short waves.

\section{A submerged bottom-standing wavemaker}

Figure 20 illustrates the effect of the gap size of type II wavemakers on the variation of the amplitude to stroke ratios versus the dimensionless water depth, $k h$. It can be seen that the curves now behave differently from those in the previous cases for type I wavemakers. The curves now seem to converge to 0 when $k h$ tends to infinity. It means that a bottomstanding wavemaker is not effective in producing high frequency surface waves, which is in contrast to type I surfacepiercing wavemakers. Moreover, it can be noted that a little increase in the gap length, as $a / h=0$ to 0.2 , reduces sharply the performance of this type of wavemakers. When $a / h$ $=0.5$, the maximum amplitude to stoke ratio is now only about 0.4; whereas a surface-piercing wavemaker of the same length and same frequency can produce waves with amplitude to stroke ratio greater than 1.5. For even shorter bottom-standing wavemakers, $a / h=0.8$ or larger, the result- ing amplitude to stroke ratios are extremely low, which has an order of only about 0.07 . Physically, surface waves are disturbances on the free surface, an oscillating plate in the bottom of the channel produces oscillatory flow near the plate with decreasing amplitude in the vertical direction. As a result, only a little bit of disturbances on the free surface are produced and thus type II wavemakers are not effective in generating surface waves.

Figure 20 also illustrates how the porosity of the plate affects the behavior of the variation of amplitude to stroke ratios vs the water depth. Not surprisingly, the porous effect in case of $a / h=0.2$ is higher than the other two case, $a / h$ $=0.5,0.8$. The spacing between the $G_{0}=0$ and $G_{0}=0.5$ curves for the case $a / h=0.2$ is larger than that in the other two cases. This can easily be explained by the fact that the length of plate in the first case, $a / h=0.2$, is longer than that in the other two cases. This is equivalent to saying that more fluid is able to pass through the porous plate and thus the porous effect is larger.

\section{A totally submerged wavemaker}

In Fig. 21, it is shown how the curves for the amplitude to stroke ratios vary as the water depth changes for different

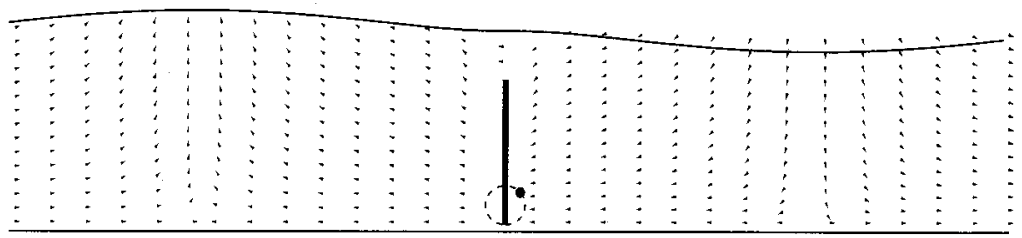

a) time $=1 / 8$ period

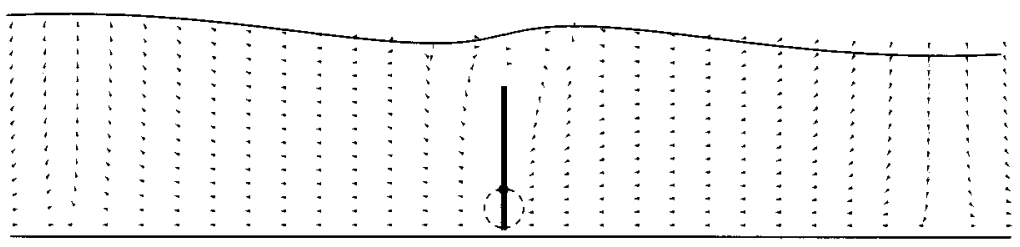

b) time $=2 / 8$ period

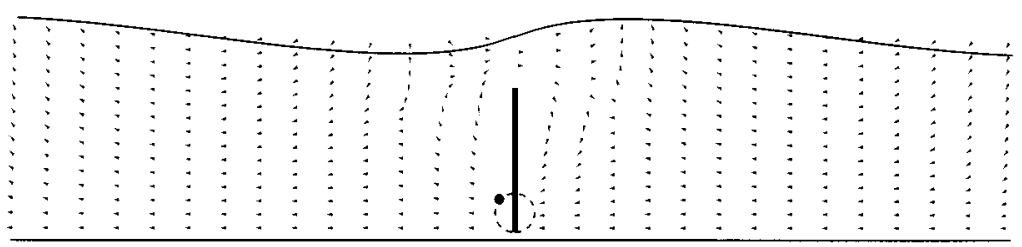

c) time $=3 / 8$ period

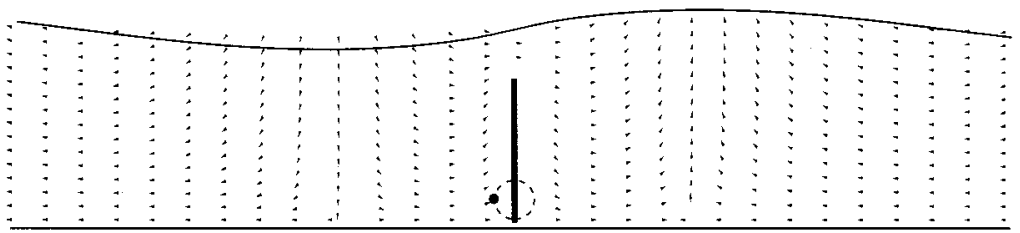

d) time $=4 / 8$ period
FIG. 22. The velocity vector field of a Type II wavemaker with $a / h=0.25, G_{0}=0$. (a) time $=1 / 8$ period; (b) time $=2 / 8$ period; (c) time $=3 / 8$ period; (d) time $=4 / 8$ period; (e) time $=5 / 8$ period; $(\mathrm{f})$ time $=6 / 8$ period; (g) time $=7 / 8$ period; (h) time $=8 / 8$ period. 


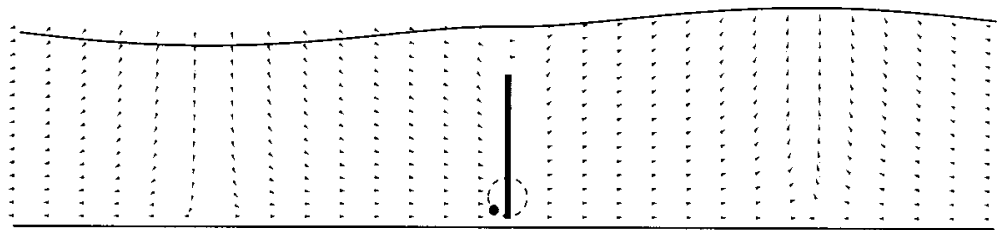

e) time $=5 / 8$ period

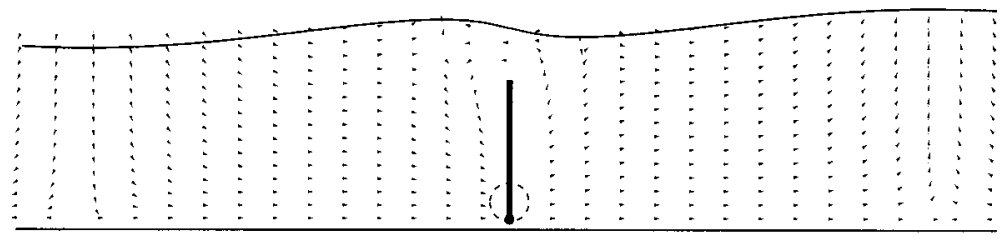

f) time $=6 / 8$ period

FIG. 22. (Continued.)

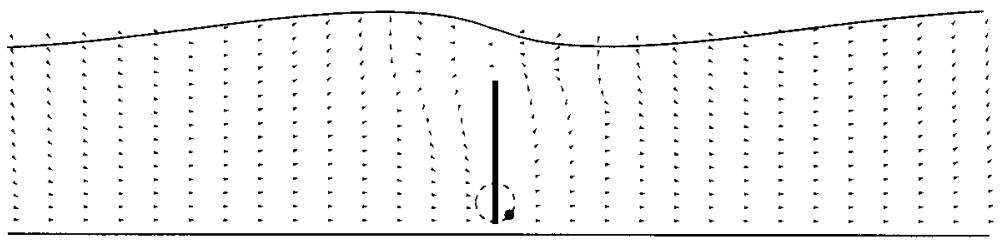

g) time $=7 / 8$ period

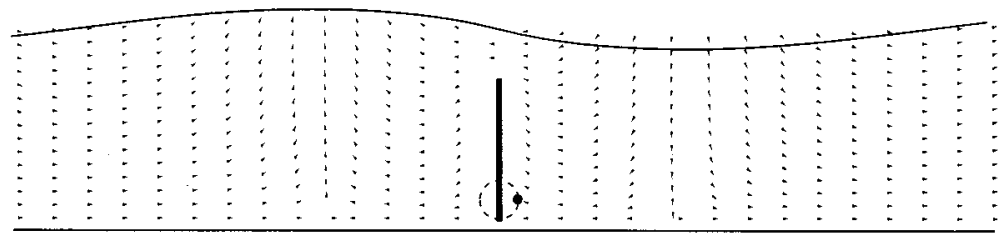

h) time $=8 / 8$ period

settings of type III impermeable wavemakers. When $a / h$ $=0$ and $d / h=1$, it represents the case of a piston-type wavemaker, which can be obtained by the classical wavemaker theory. The second curve with $a / h=0.25$ and $d / h=0.75$ actually resembles a type II wavemaker, so the general shape of this curve is similar to those shown in Fig. 20. For the third curve with $a / h=0.25$ and $d / h=0.5$, the values of the amplitude to stroke ratios are generally much lower than those of the former case with $a / h=0.25$ and $a / d=0.75$. This result is reasonable, as the length of the wavemaker is now smaller. If the length of the plate is further reduced, the amplitude to stroke ratios get even smaller as shown in the curves with $a / h=0.25$ and $d / h=0.25$. It should be noted that the curves for the cases, $a / h=0.25$ and $d / h=0.5, a / h$ $=0.25$ and $d / h=0.25, a / h=0.5$ and $d / h=0.25$ seem to be flatter than that for the case with $a / h=0.25$ and $d / h=0.75$.

Figure 21 also demonstrates the porous effect on the variation of amplitude to stroke ratios versus water depth for different settings of type III wavemakers. Consider the case when the positions of the plates are fixed at $a / h=0.25$, while the length of the plate is decreased from $d / h=0.5$ to $d / h$ $=0.25$. As discussed earlier, decreasing the length of the wavemaker has two effects, the first one is to reduce the amplitude to stroke ratios and the second one is to reduce the degree of the porous effect. These results can be observed in those curves for the two cases. The values for the curve $G_{0}$ $=0, a / h=0.25$ and $d / h=0.5$ are higher than those for the case with $a / h=0.25$ and $d / h=0.25$. Also, the other curves for the case with $a / h=0.25$ and $d / h=0.5$ are more scattered than those for the case with $a / h=0.25$ and $d / h=0.25$.

Consider another case when the length of the wavemaker is fixed at $d / h=0.25$, while the positions of the plates are changed from $a / h=0.25$ to $a / h=0.5$. Shifting the plate downwards seems also to have two effects, the first one is to reduce the amplitude to stroke ratios and the second one is to reduce the effectiveness in generating high frequency waves. These are shown by two observations in the curves for the corresponding two cases. The values for the curves for the case with $a / h=0.25$ and $d / h=0.25$ are much larger than those for the case with $a / h=0.5$ and $d / h=0.25$. Also, the curves for the case with $a / h=0.5$ and $d / h=0.25$ seem to drop much earlier than those for the case with $a / h=0.25$ and $d / h=0.25$.

\section{CONCLUSIONS}

The problems of scattering and radiation of surface waves by several types of permeable vertical porous barriers 
are studied for finite water depth. The variation of certain useful parameters versus the dimensionless water depth has been obtained by applying the method of eigenfunction expansions and a suitable application of the least squares method. There is a strong relation between the scattering and radiation problems. Specifically, it is noted that for type-I barriers and type-I wavemakers the reflection coefficient and the amplitude-to-stroke ratio give the same limiting ratio as $k h$ tends to infinity for different values of $G_{0}$. For example, the reflection coefficient for $G_{0}=0.5$ is half of that for $G_{0}$ $=0$ as $k h$ approaches infinity (see Fig. 6), and the amplitudeto-stroke ratio for $G_{0}=0.5$ is also half of that for $G_{0}=0$ as $k h$ tends to infinity (see Fig. 18). Therefore, it is possible to determine the porosity of a plate by measuring the amplitude-to-stroke ratio which is practically easier to obtain.

It should be noted that although only real values of the Chwang parameter $G_{0}$ have been considered in this paper, it is possible to use complex values of $G_{0}\left(\right.$ see $\mathrm{Yu}^{27}$ ). However, for a medium in which the resistance dominates the inertial effect, like the present study, the porous parameter $G_{0}$ becomes purely real.

Moreover, the solution obtained by the present method is a full solution. Many useful information can further be calculated from the solution, for example, the wave force, the wave moment and the velocity vector field as shown in Fig. 22 (in which the circle and dot represent the phase of the moving plate).

Based on the results obtained, a porous barrier can reduce the impinging hydrodynamic wave forces acting on it and, at the same time, maintain a reasonably good performance when it is used as a breakwater or wavemaker.

\section{ACKNOWLEDGMENT}

This research was sponsored by the Hong Kong Research Grants Council under Grant No. HKU 568/96E.

${ }^{1}$ F. Ursell, "The effect of a fixed vertical barrier on surface waves in deep water," Proc. Cambridge Philos. Soc. 43, 374 (1947).

${ }^{2}$ F. Ursell, "On the waves due to the rolling of a thin strip," Q. J. Mech. Appl. Math. 1, 246 (1948).

${ }^{3}$ W. R. Dean, "On the reflection of surface waves by a submerged plane barrier,' Proc. Cambridge Philos. Soc. 41, 231 (1945).

${ }^{4}$ W. E. Williams, "Note on the scattering of water waves by a vertical barrier,"' Proc. Cambridge Philos. Soc. 62, 507 (1966).

${ }^{5}$ D. V. Evans, "Diffraction of water waves by a submerged vertical plate," J. Fluid Mech. 40, 433 (1970).

${ }^{6} \mathrm{E}$. O. Tuck, "Transmission of water waves through small apertures," J. Fluid Mech. 49, 65 (1971).

${ }^{7}$ D. Porter, "The transmission of surface waves through a gap in a vertical barrier," Proc. Cambridge Philos. Soc. 71, 411 (1972).

${ }^{8}$ J. J. Stoker, Water Waves (Interscience, New York, 1957).

${ }^{9} \mathrm{H}$. Levine and E. Rodemich, "Scattering of surface waves on an ideal fluid," Technical Report No. 78, Math. and Stat. Lab., Stanford University, CA, 1958.

${ }^{10} \mathrm{M}$. Lewin, "The effect of vertical barriers on progressive waves," J. Math. Phys. 42, 287 (1963).

${ }^{11} \mathrm{C}$. C. Mei, "Radiation and scattering of transient gravity waves by vertical plates," Q. J. Mech. Appl. Math. 19, 417 (1966).

${ }^{12}$ P. L.-F. Liu and M. Abbaspour, "Wave scattering by a rigid thin barrier," J. Wtwy., Port, Coast. and Ocean Engrg., ASCE 108, 479 (1982).

${ }^{13}$ I. J. Losada, M. A. Losada, and A. J. Roldan, "Propagation of oblique incident waves past rigid vertical thin barriers," Appl. Ocean Res. 14, 191 (1992).

${ }^{14}$ A. G. Abul-Azm, "Wave diffraction through submerged breakwaters," J. Wtwy., Port, Coast. and Ocean Engrg., ASCE 119, 587 (1993).

${ }^{15}$ R. Porter and D. V. Evans, "Complementary approximations to wave scattering by vertical barriers," J. Fluid Mech. 294, 155 (1995).

${ }^{16} \mathrm{D}$. V. Evans, "A note on the waves produced by small oscillations of a partially immersed vertical plate," J. Inst. Math. Appl. 17, 135 (1976).

${ }^{17}$ D. V. Evans and C. M. Linton, "Active devices for the reduction of wave intensity,” Appl. Ocean Res. 11, 26 (1989).

${ }^{18}$ D. V. Evans and R. Porter, "Hydrodynamic characteristics of a thin rolling plate in finite depth of water," Appl. Ocean Res. 18, 215 (1997).

${ }^{19} \mathrm{C}$. Macaskill, "Reflexion of water waves by a permeable barrier," J. Fluid Mech. 95, 141 (1979).

${ }^{20}$ A. T. Chwang, "A porous wavemaker theory," J. Fluid Mech. 132, 395 (1983).

${ }^{21}$ A. T. Chwang and A. T. Chan, "Interaction between porous media and wave motion," Annu. Rev. Fluid Mech. 30, 53 (1998).

${ }^{22}$ G. I. Taylor, "Fluid flow in regions bounded by porous surfaces," Proc. R. Soc. A 234, 456 (1956).

${ }^{23}$ I. N. Sneddon, Mixed Boundary Value Problems in Potential Theory (North-Holland, Amsterdam, 1966).

${ }^{24}$ R. B. Kelman and C. A. Koper, "Least squares approximations for dual trigonometric series," Glasgow Math. J. 14, 111 (1973).

${ }^{25}$ R. P. Feinerman and R. B. Kelman, "The convergence of least squares approximations for dual orthogonal series," Glasgow Math. J. 15, 82 (1974)

${ }^{26}$ A. T. Chwang and Z. Dong, "Wave-trapping due to a porous plate," in Proceedings of the 15th Symposium on Naval Hydrodynamics (National Academy Press, 1985), p. 407.

${ }^{27} \mathrm{X}$. Yu, "Diffraction of water waves by porous breakwaters," J. Wtwy., Port, Coast. and Ocean Engrg., ASCE 121, 275 (1995). 